\title{
Geleitwort von Pascal Strupler
}

Was auch immer der Antrieb sein mag, Medizin zu studieren oder sich einem Gesundheitsberuf hinzuwenden - früher oder später wird sich Ihr Tätigkeitsfeld einengen - auf ein Spezialgebiet, auf eine Altersgruppe, auf ein einziges Organ, eine einzelne Krankheit. Selbst wenn Sie sich der Breite der Hausarztmedizin zuwenden sollten, wird immer ein größerer oder kleinerer Teil des Gesundheitswesens im toten Winkel Ihres Blickfeldes liegen.

Der Trend $\mathrm{zu}$ thematischer Fokussierung bringt gewiss große und notwendige Fortschritte auf jedem Gebiet, auch in der Medizin. Er ist aber eng verwoben mit den Gefahren jeder monothematischen Ausrichtung: Zusammenhänge werden leicht übersehen, kritische Fragen überhört, Ursachen am falschen Ort gesucht, wichtige Einflüsse unterschätzt.

Umso notwendiger ist - gerade beim Einstieg in die medizinische Berufsausbildung - eine Rundumsicht auf das, was Gesundheit ausmacht und Krankheit zu vermeiden vermag, auf die Versorgung der Gesamtbevölkerung mit gesundheitsfördernden oder -erhaltenden Diensten. Es ist das große Verdienst dieses Buches, einen strukturierten, stringenten und spannenden Einblick in die Welt der öffentlichen Gesundheitsbelange zu vermitteln und zur Vertiefung breiterer Kenntnisse anzuregen.

Public Health steht für Gesundheitsbestreben im weiteren, gesellschaftlichen, gesellschaftspolitischen Sinn. Sie steht auch für die Erkenntnis in die Notwendigkeit zur Zusammenarbeit aller beteiligten Akteure im Interesse der öffentlichen Gesundheit. Damit richtet sich das Buch selbstverständlich nicht nur an das klassische Gesundheitspersonal. Public Health ist - hoffentlich - auch der Anspruch der Gesundheitspolitik auf optimale Berücksichtigung der Gesundheit in allen Bereichen der Politik. Insofern ist die Lektüre auch für all jene empfehlenswert, die sich mit dem Blick aufs Ganze im gesundheitspolitischen Umfeld bewegen, oder dort etwas bewegen wollen.

\section{Pascal Strupler}

Ehem. Direktor des Bundesamtes für Gesundheit

Bern 
